\title{
Enhanced Next Generation Millimeter-Wave Multicarrier System with Generalized Frequency Division Multiplexing
}

\author{
Hidekazu Shimodaira, ${ }^{1}$ Joongheon Kim, ${ }^{2}$ and Ali S. Sadri ${ }^{3}$ \\ ${ }^{1}$ NTT DOCOMO, Inc., Tokyo, Japan \\ ${ }^{2}$ School of Computer Science and Engineering, Chung-Ang University, Seoul, Republic of Korea \\ ${ }^{3}$ mmWave Standards and Advanced Technology (mSAT) Team, Intel Corporation, San Diego, CA, USA
}

Correspondence should be addressed to Joongheon Kim; joongheon@gmail.com

Received 25 July 2016; Revised 7 November 2016; Accepted 14 November 2016

Academic Editor: Imdad Khan

Copyright (C) 2016 Hidekazu Shimodaira et al. This is an open access article distributed under the Creative Commons Attribution License, which permits unrestricted use, distribution, and reproduction in any medium, provided the original work is properly cited.

\begin{abstract}
Orthogonal Frequency Division Multiplexing (OFDM) is a popular multicarrier technique used to attain high spectral efficiencies. It also has other advantages such as multipath tolerance and ease of implementation. However, OFDM based systems suffer from high Peak-to-Average Power Ratio (PAPR) problem. Because of the nonlinearity of the power amplifiers, the high PAPR causes significant distortion in the transmitted signal for millimeter-wave (mmWave) systems. To alleviate the high PAPR problem, this paper utilizes Generalized Frequency Division Multiplexing (GFDM) which can achieve high spectral efficiency as well as low PAPR. In this paper, we show the performance of GFDM using the IEEE 802.11ad multicarrier frame structures. IEEE 802.11ad is considered one of the most successful industry standards utilizing unlicensed mmWave frequency band. In addition, this paper indicates the feasibility of using GFDM for the future standards such as IEEE 802.11ay. This paper studies the performance improvements in terms of PAPR reduction for GFDM. Based on the performance results, the optimal numbers of subcarriers and subsymbols are calculated for PAPR reduction while minimizing the Bit Error Rate (BER) performance degradation. Moreover, transmitter side ICI (Intercarrier Interference) reduction is introduced to reduce the receiver load.
\end{abstract}

\section{Introduction}

The demands for wireless data communications have been increasing year by year. Recently, transmitting pictures or movies through a wireless channel is very popular and even $4 \mathrm{~K}$ resolution ultra-high definition (UHD) video can be transmitted in real-time over the wireless channel. In order to support this kind of massive wireless data transmissions, ultra-wide channel bandwidths are essentially required. Among various candidates, millimeter-wave (mmWave) wireless communication technology is one of promising ultra-wideband wireless communication technologies in order to realize multi-gigabit-per-second (multi-Gbps) ultrahigh speed wireless data communications. For instance, $60 \mathrm{GHz}$ mmWave channels have a bandwidth of $2.16 \mathrm{GHz}$ [1].

Among several mmWave wireless technologies, this paper mainly focuses on $60 \mathrm{GHz}$ IEEE 802.11ad as it is the most successful standard in mmWave research societies $[1,2]$. However, the proposed methodologies are applicable for other
mmWave standards including $28 \mathrm{GHz}$ and $38 \mathrm{GHz}$ mmWave frequency bands [3-6]. The IEEE 802.11ad (hereinafter referred to as 1lad) is one of the mmWave wireless communication systems which uses $60 \mathrm{GHz}$ ISM (Industry-Science-Medical) band. In more detail, $57-66 \mathrm{GHz}$ is allocated to the $60 \mathrm{GHz}$ ISM band and 11ad separates this $9 \mathrm{GHz}$ band into four channels of $2.16 \mathrm{GHz}$ bandwidth each. 11ad supports two types of transmission schemes, Single Carrier (SC) and Orthogonal Frequency Division Multiplexing (OFDM) transmission, and theoretical maximum throughput reaches about 7 Gbps using the OFDM transmission scheme. OFDM efficiently utilizes system bandwidth based on (i) subcarrier orthogonality and (ii) intersymbol interference (ISI) mitigation due to the narrow band characteristics in each subcarrier. However, parallel transmission using large number of subcarriers increases Peak-to-Average Power Ratio (PAPR) [7-9]. In 60 GHz communication systems, the demand for power amplifier is more severe than that of microwave systems and hence high PAPR 
is undesirable. In order to tackle this problem there are two major approaches: (i) applying PAPR reduction techniques for OFDM and (ii) introducing new waveforms.

The first approach, that is, the PAPR reduction techniques for OFDM, has been studied extensively in the literature. One such PAPR reduction technique is transmit signal clipping [10-12]. This technique sets a threshold value and just clips the high amplitudes. Since some transmit signals are distorted and undesired signals are radiated by clipping, the clipped signals are filtered before transmission. The other commonly used PAPR reduction technique is signal companding. This technique applies nonlinear compression to the transmit signals to suppress the signal peak values [13-15]. Partial Transmit Sequences (PTS) technique [16-18] divides subcarriers into segments and modulates each block. After modulation, phase rotation factor is applied to each partial sequence in time domain, and the subsegments are combined. Selected Mapping (SLM) technique makes several OFDM modulated signals where each OFDM modulation has its own different phase weight and chooses the best one from candidates [1921].

The second approach, that is, new waveform design for the next generation wireless systems, has been currently investigated actively. From the view point of PAPR reduction, Filter Bank Multicarrier (FBMC) is one of the well-investigated waveforms [22]. FBMC applies pulse shaping filter to each subcarrier. The filter bank structure can be adapted to the clipping technique $[23,24]$. However since clipping technique induces not only the out-of-band radiation but also the inband distortion, the system becomes more complicated. Generalized Frequency Division Multiplexing (GFDM) [25, 26] is also one of the new waveform candidates and it can reduce PAPR efficiently utilizing more straightforward methods. GFDM can flexibly configure the symbol structure; that is, the number of subcarriers can be configured by introducing time domain slicing. Since GFDM can have the characteristics of both single carrier and multicarrier transmission, low PAPR and high spectral efficiency can be achieved simultaneously by applying appropriate configuration.

This paper focuses on GFDM and proposes GFDM frame design for llad to be fully in compliance with standards. The validity of the proposed frame structure is shown through intensive numerical simulations. Based on the PAPR and Bit Error Rate (BER) simulation results, the best GFDM configuration for 1lad system is determined. The computational complexity of GFDM is analyzed and a low complexity modulator/demodulator is proposed. Using the scheme proposed in this paper, the performance of current multicarrier mmWave standards can be improved in terms of the throughput for various mmWave systems such as backhaul in line-of-sight (LOS), fronthaul in LOS, and access networks in both LOS and non-LOS (NLOS).

The remaining sections are organized as follows. Section 2 explains OFDM and GFDM briefly and introduces the llad OFDM structure. Section 3 proposes a new GFDM frame structure for 1lad. Section 4 introduces a novel low complexity interference canceller for GFDM. PAPR and BER comparison is shown in Section 5. Section 6 analyzes the computational complexity of modulation/demodulation architectures. Finally, conclusion and future work are provided in Section 7.

\section{System Overview}

In this section, we first discuss the OFDM and GFDM symbol structures and then describe the 1lad OFDM system architecture.

2.1. Overview of OFDM. OFDM is one of the well-known multicarrier transmission schemes [27-31]. It is widely employed by several wireless systems including Digital Terrestrial Television Broadcasting, LTE (Long Term Evolution), and IEEE $802.11 \mathrm{a} / \mathrm{g} / \mathrm{n} / \mathrm{ac} / \mathrm{ad}$. Since OFDM transmits signals in parallel utilizing many subcarriers, each subcarrier bandwidth becomes narrow and thus the ISI is mitigated. Moreover, thanks to the FFT (Fast Fourier Transform) algorithm in OFDM structures, computational and implementation costs can be reduced.

The mathematical expression of an OFDM transmission symbol is as follows;

$$
x[n]=\sum_{k=0}^{N-1} d_{k} \exp \left(j 2 \pi \frac{n k}{N}\right),
$$

where $x[n]$ is the transmission symbol of $n$th time sample and $k$ and $N$ are the subcarrier index and the total number of subcarriers, respectively. $d_{k}$ in (1) is the QAM (Quadrature Amplitude Modulation) modulated symbol transmitted on the $k$ th subcarrier. Equation (1) describes parallel transmission of $N$ modulated symbols $\left(d_{0}, d_{1}, \ldots, d_{N-1}\right)$ using $N$ orthogonal subcarriers. Please note that (1) is equivalent to the definition of IDFT (Inverse Discrete Fourier Transform), and hence the transmitted data symbols in (1) can be rewritten as follows:

$$
\begin{aligned}
& \mathbf{x}=\mathbf{F}^{\mathrm{H}} \mathbf{d} \\
& \mathbf{x}=\left[\begin{array}{llll}
x[0] & x[1] & \cdots & x[N-1]
\end{array}\right]^{\mathrm{T}} \in \mathbb{C}^{N} \\
& \mathbf{F}=\left[\begin{array}{cccc}
w_{N}^{0 \cdot 0} & w_{N}^{0 \cdot 1} & \cdots & w_{N}^{0 \cdot(N-1)} \\
w_{N}^{1 \cdot 0} & w_{N}^{1 \cdot 1} & \cdots & w_{N}^{1 \cdot(N-1)} \\
\vdots & \vdots & \ddots & \vdots \\
w_{N}^{(N-1) \cdot 0} & w_{N}^{(N-1) \cdot 1} & \cdots & w_{N}^{(N-1) \cdot(N-1)}
\end{array}\right] \in \mathbb{C}^{N \times N} \\
& \mathbf{d}=\left[\begin{array}{llll}
d_{0} & d_{1} & \cdots & d_{N-1}
\end{array}\right]^{\mathrm{T}} \in \mathbb{C}^{N},
\end{aligned}
$$

where $w_{N}=\exp (-j(2 \pi / N))$ is a primitive $N$ th root of unity. $(\cdot)^{\mathrm{T}}$ and $(\cdot)^{\mathrm{H}}$ represent transpose and conjugate transpose, respectively. Figure 1 shows how to map modulated symbols on the time-frequency resource grid in OFDM.

On the receiver side, received signals can be demodulated using DFT operation.

2.2. Overview of GFDM. As the name suggests, GFDM generalizes the multicarrier modulation schemes, SC and OFDM, 


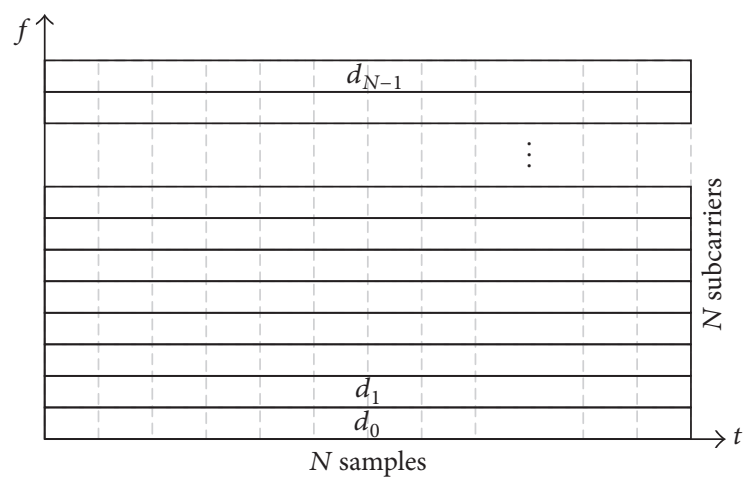

FIGURE 1: OFDM symbol mapping structure.

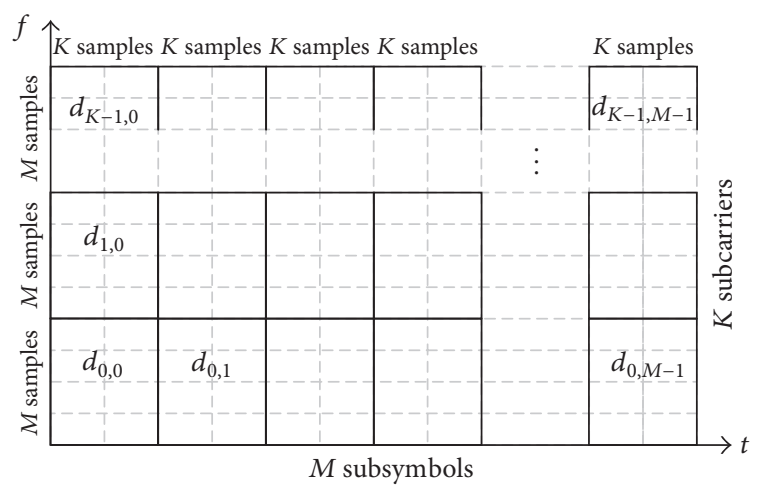

Figure 2: GFDM symbol mapping structure.

by controlling the system design parameters. In GFDM, a time-frequency resource grid is sliced in both frequency and time domains. QAM modulated symbols are mapped on each time-frequency resource block. Figure 2 shows the mapping illustration of GFDM where $K$ and $M$ stand for the number of subcarriers and number of subsymbols, respectively. Each resource block has $K \times M$ sample points. Therefore if $K \times M=$ $N$ is satisfied, the amount of transmission data with GFDM will be equivalent to the amount of transmission data with OFDM during same symbol duration in same bandwidth. In order to localize each resource block, pulse shaping filter is applied.

The mathematical expression of GFDM transmission signal is as follows:

$$
\begin{aligned}
x[n]= & \sum_{k=0}^{K-1} \sum_{m=0}^{M-1}\left(d_{k, m} \delta[n-m K]\right) \\
& * g[n \bmod N] \exp \left(j 2 \pi \frac{n k}{K}\right) \\
= & \sum_{k=0}^{K-1} \underbrace{\sum_{m=0}^{M-1} d_{k, m} \tilde{g}[n-m K]}_{x_{k}[n]} \exp \left(j 2 \pi \frac{n k}{K}\right) \\
= & \sum_{k=0}^{K-1} x_{k}[n] \exp \left(j 2 \pi \frac{n k}{K}\right),
\end{aligned}
$$

where $(*)$ represents the convolution operation. In (3), $\tilde{g}[n-m K] \triangleq g[(n-m K) \bmod N]$ is the pulse shaping filter with $m K$ time shifting. The modulo operation in (3) is equivalent to the tail biting process which makes filter convolution circular [32]. The GFDM modulation procedure is enumerated below and also shown in Figure 3.

(1) Symbol sequence is divided into $K$ parallel blocks, with each containing $M$ QAM modulated symbols.

(2) The QAM modulated symbol $d_{k, m}$ is upsampled by a factor $N$.

(3) Pulse shaping filter is applied and the subsymbols are summed up in time domain.

(4) Filtered symbols are upconverted to the corresponding subcarrier frequency.

(5) All symbols are summed up.

In case of $M=1$ and rectangular pulse shaping filter, (3) reduces to (1). In case of $K=1,(3)$ is equivalent to $\mathrm{SC}$ transmission. For this reason, this scheme is denoted as GFDM.

Equation (3) can be rewritten as a linear equation $\mathbf{x}=$ Ad. The matrix $\mathbf{A}$ and vector $\mathbf{d}$ are as follows:

$$
\begin{aligned}
& \mathbf{A}=\left[\begin{array}{llll}
\mathbf{G}_{0} \circ \mathbf{W}_{K} & \cdots & \mathbf{G}_{M-1} \circ \mathbf{W}_{K}
\end{array}\right] \in \mathbb{C}^{N \times K M} \\
& \mathbf{G}_{m}=\underbrace{\left[\begin{array}{llll}
\widetilde{\mathbf{g}}_{m} & \widetilde{\mathbf{g}}_{m} & \cdots & \widetilde{\mathbf{g}}_{m}
\end{array}\right]}_{K} \in \mathbb{C}^{N \times K}
\end{aligned}
$$

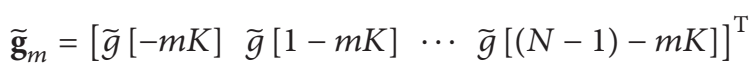

$$
\begin{aligned}
& \in \mathbb{C}^{N} \\
& \mathbf{W}_{K}=\left[\begin{array}{cccc}
w_{K}^{0 \cdot 0} & w_{K}^{0 \cdot 1} & \cdots & w_{K}^{0 \cdot(K-1)} \\
w_{K}^{1 \cdot 0} & w_{K}^{1 \cdot 1} & \cdots & w_{K}^{1 \cdot(K-1)} \\
\vdots & \vdots & \ddots & \vdots \\
w_{K}^{(N-1) \cdot 0} & w_{K}^{(N-1) \cdot 1} & \cdots & w_{K}^{(N-1) \cdot(K-1)}
\end{array}\right] \in \mathbb{C}^{N \times K} \\
& \mathbf{d}=\left[\begin{array}{lllll}
\mathbf{d}_{0}^{\mathrm{T}} & \cdots & \mathbf{d}_{m}^{\mathrm{T}} & \cdots & \mathbf{d}_{M-1}^{\mathrm{T}}
\end{array}\right]^{\mathrm{T}} \in \mathbb{C}^{K M} \\
& \mathbf{d}_{m}=\left[\begin{array}{llll}
d_{0, m} & d_{1, m} & \cdots & d_{K-1, m}
\end{array}\right]^{\mathrm{T}} \in \mathbb{C}^{K},
\end{aligned}
$$

where the operation ( $\circ$ ) represents the Hadamard product.

This paper assumes that the receiver employs correlation detector. The correlation receiver is realized by multiplying $\mathbf{A}^{\mathrm{H}}$ to the received GFDM symbols. Since $\mathbf{A}$ contains upconversion term for each subcarrier, downconversion can be performed through $\mathbf{A}^{\mathrm{H}}$ operation. Figure 4 shows normalized absolute value of $\mathbf{A}^{\mathrm{H}} \mathbf{A}$. As shown in the figure, intercarrier interference (ICI) appears due to the nonorthogonality of subcarriers.

2.3. WiGig/IEEE 802.11ad OFDM Frame Structure. The 1lad OFDM frame is composed of five fields as defined in the standard, that is, Short Training Field (STF), Channel Estimation Field (CEF), Header, OFDM symbols, and optional training 


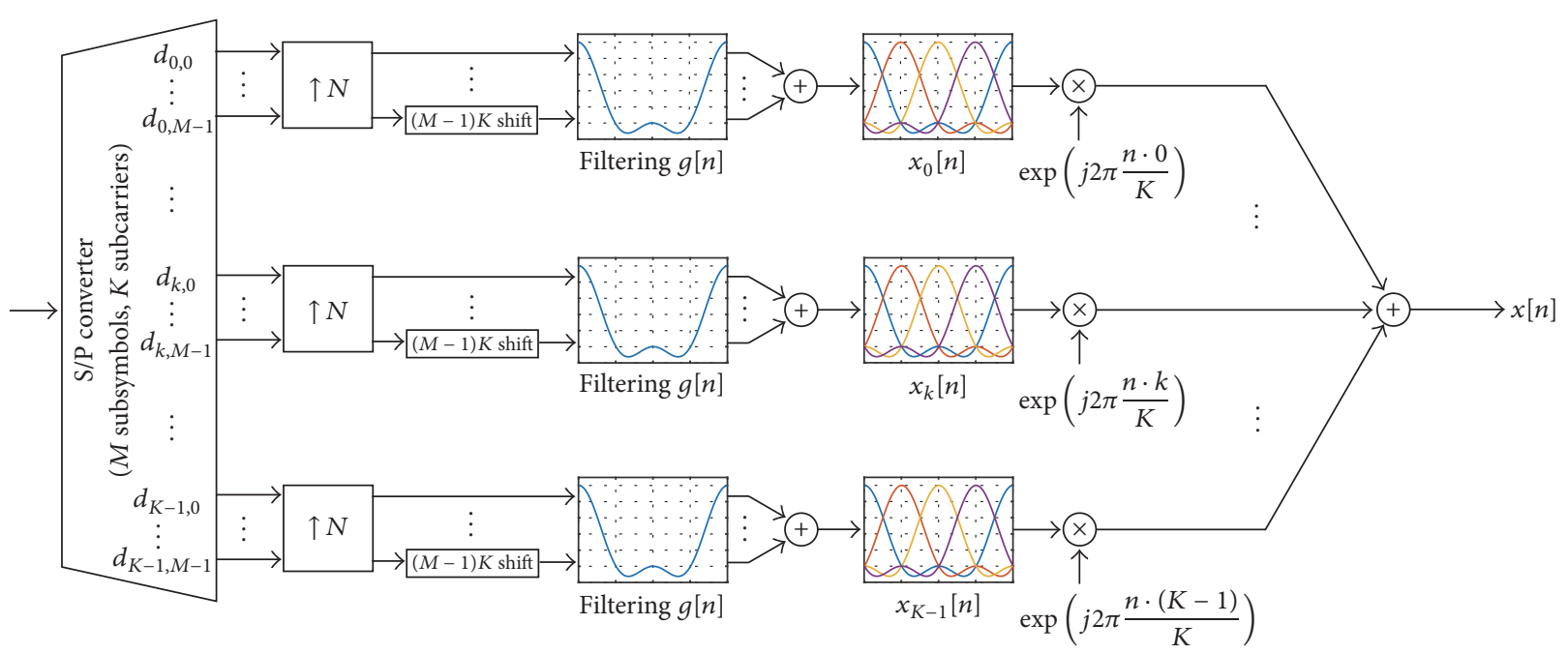

FIGURE 3: GFDM modulation block diagram.

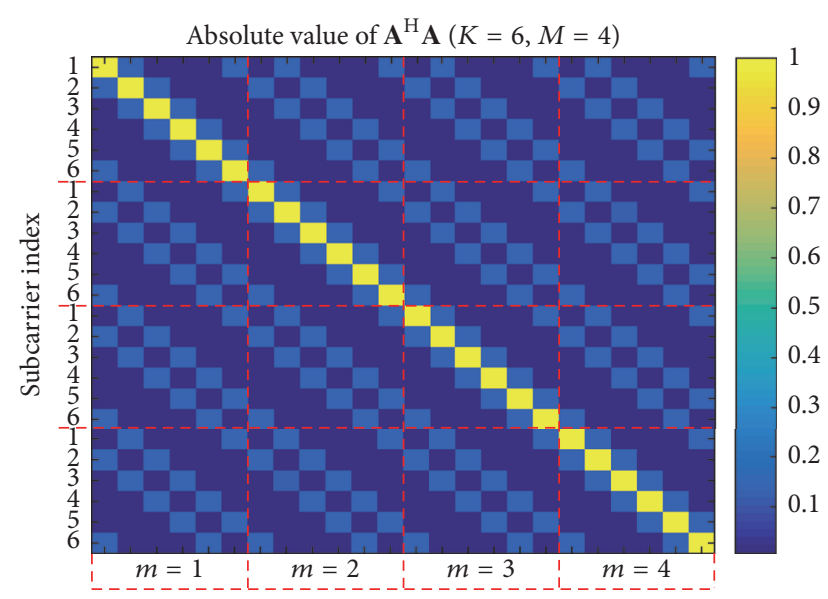

FIGURE 4: ICI effect of the GFDM (Root Raised Cosine filter with roll-off factor 0.1. $K=6, M=4$ ).

fields. Since the header and optional training fields do not impact the performance, these fields are ignored in this paper.

The STF is used for the carrier frequency offset (CFO) estimation and autogain control (AGC) configuration. After CFO compensation and AGC, propagation channels are estimated using CEF. These two fields use Golay complementary sequence with $\pi / 2$-shift BPSK modulation [33]. Figure 5 shows the STF and CEF structures of control PHY (for control signal transmission), SC PHY, and OFDM PHY.

$\mathrm{Ga}_{128}$ and $\mathrm{Gb}_{128}$ in Figure 5 stand for 128-bit Golay complementary sequences. $\mathrm{Gu}_{512}$ and $\mathrm{Gv}_{512}$ are not the 512-bit Golay complementary sequences; however they are constructed with the concatenation of $\mathrm{Ga}_{128}$ and $\mathrm{Gb}_{128}$. The 1lad receiver can distinguish each frame due to the difference between STF and CEF. The last $-\mathrm{Ga}_{128}$ in STF indicates the end of STF or the start point of CEF.

The OFDM symbol follows these preamble fields. The number of OFDM symbols within one packet varies according to the transmitted data and MCS (Modulation and
TABlE 1: WiGig/IEEE 802.11ad OFDM parameters.

\begin{tabular}{lc}
\hline Parameter & Value \\
\hline $\begin{array}{l}\text { Number of IDFT/DFT } \\
\text { points }\end{array}$ & 512 \\
$\begin{array}{l}\text { Number of data } \\
\text { subcarriers }\end{array}$ & 336 \\
$\begin{array}{l}\text { Number of pilot } \\
\text { subcarriers }\end{array}$ & $16( \pm 10,30,50,70,90,110,130,150)$ \\
$\begin{array}{l}\text { Number of DC } \\
\text { subcarriers }\end{array}$ & $3(-1,0,1)$ \\
$\begin{array}{l}\text { Number of guard band } \\
\text { subcarriers }\end{array}$ & $157(-256 \sim-178,178 \sim 255)$ \\
Subcarrier frequency & \\
spacing & $5.15625 \mathrm{MHz}(2640 \mathrm{MHz} / 512)$ \\
OFDM sample rate & $2640 \mathrm{MHz}$ \\
OFDM symbol period & $0.194 \mathrm{usec}$ \\
Cyclic prefix duration & $48.4 \mathrm{~ns}=0.194 \mathrm{usec} \times 25 \%$ \\
\hline
\end{tabular}

Coding Scheme). Table 1 shows important parameter configurations and corresponding values of 1lad OFDM. This paper proposes the GFDM frame structure for 1lad based on these parameters.

\section{GFDM for IEEE 802.11ad Systems}

GFDM has a potential to reduce the PAPR while achieving high throughput performance. However, in order to utilize GFDM in 1lad, transmission system has to be in compliance with the standard. This section proposes the designs of GFDM spectrum and pilot symbols.

3.1. GFDM DC Null and Guard Band Design. If there are no constraints, multicarrier transmission systems use entire bandwidth by separating several subcarriers. However if the "transmission system" is considered, DC subcarrier and guard band subcarrier have to be nullified to avoid DC offset 


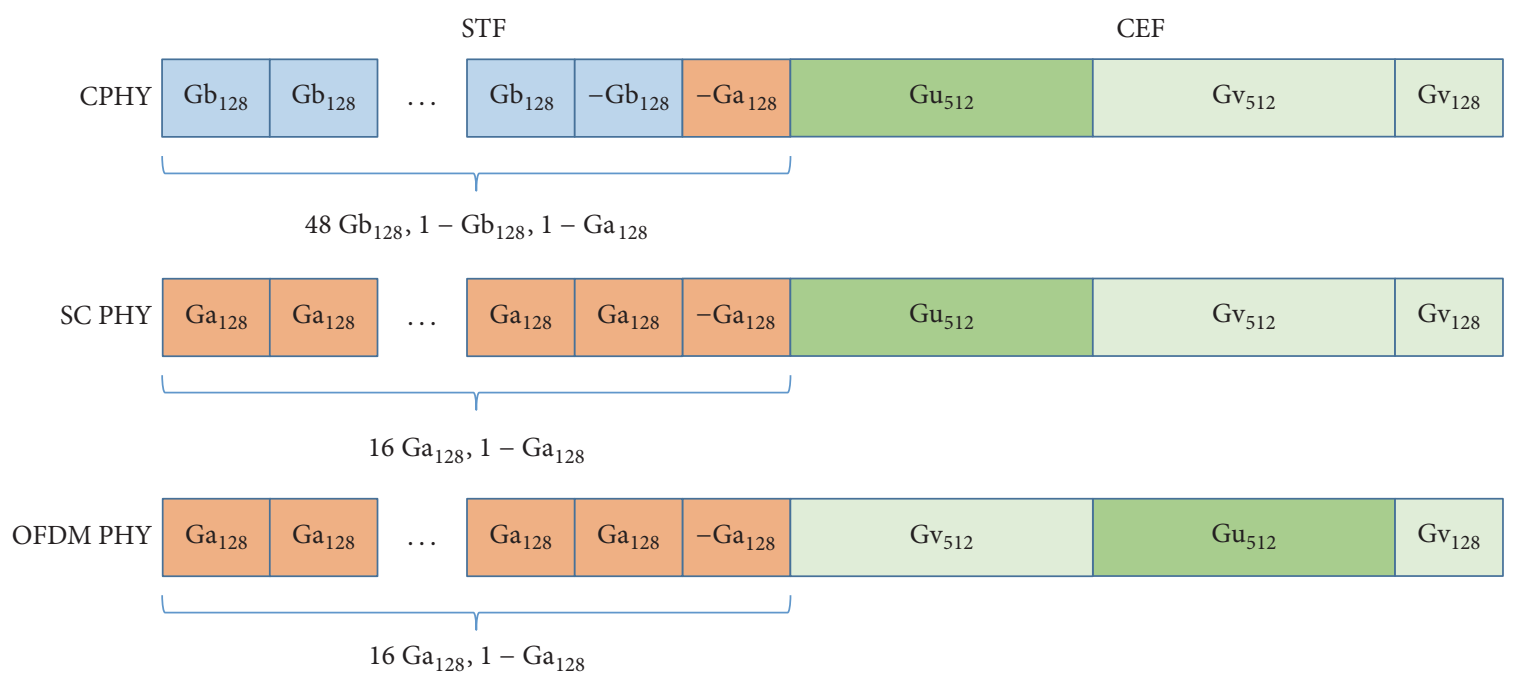

FIGURE 5: WiGig/IEEE 802.11ad preamble structure.

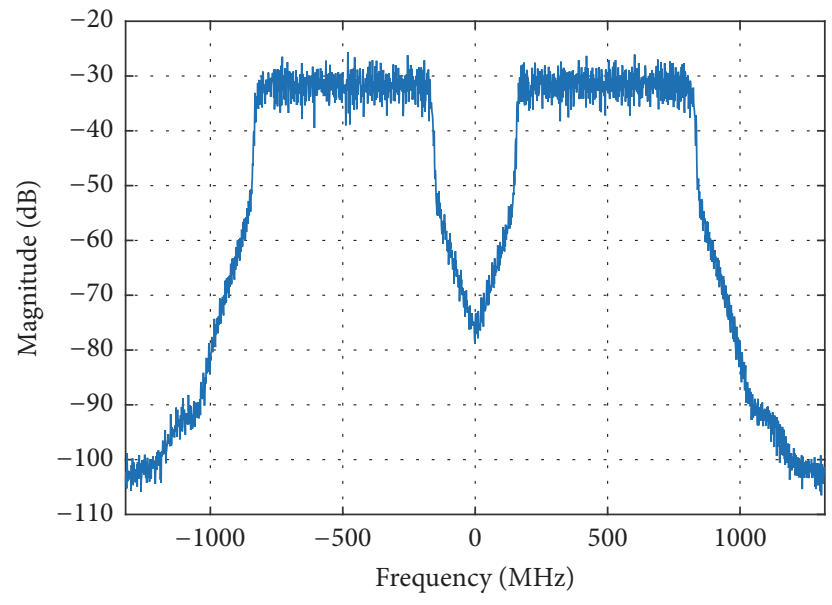

Figure 6: GFDM spectrum with DC nulling $(K=8, M=64)$.

and interference for other channels or other systems. Based on Table 1, the GFDM transmission system for llad is designed.

Since GFDM uses small number of subcarriers to reduce PAPR, the subcarrier bandwidth becomes larger than that of OFDM. In the case of OFDM, DC nulling and guard band insertion are just setting zero to the corresponding subcarriers. In the GFDM case, this method results in the waste of frequency resources. Figure 6 shows the spectrum of conventional DC nulling and guard band insertion in the case of $K=8$. Since the subcarrier spacing in this case is $330 \mathrm{MHz}(2640 \mathrm{MHz} / 8)$, DC null is very wide, and therefore fine-adjustment of guard bands is impossible. In addition, additional guard band insertion is required because this spectrum cannot satisfy 1lad requirement. Consequently, frequency resources are wasted.

This paper proposes subcarrier upshifting/downshifting methods to avoid the wasting of resources. As shown in Figure 3, GFDM modulation has upconversion blocks at

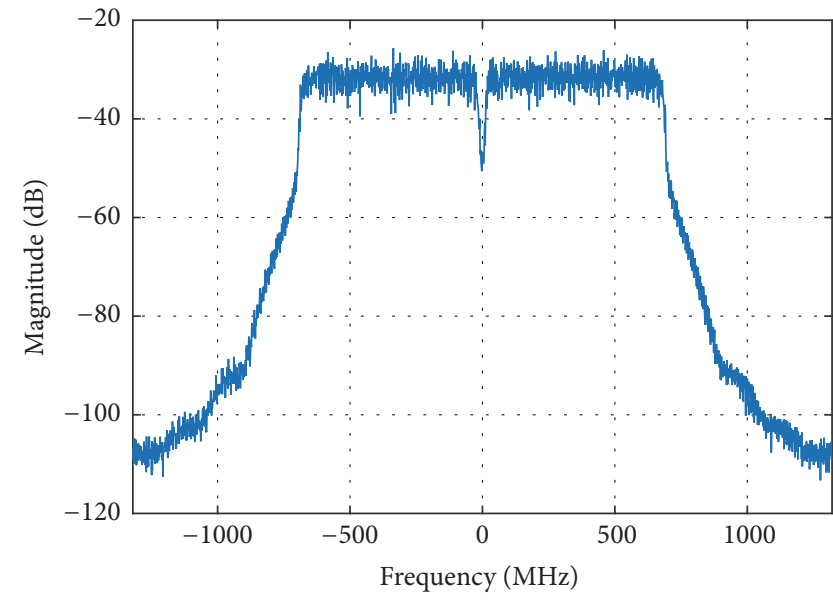

FIGURE 7: GFDM spectrum with DC nulling and frequency shift $(K=8, M=64)$.

the last part. By introducing additional frequency shifting block, wide DC null can be shrunk. This can be done by downshifting the lower side $(k=0,1, \ldots, K / 2)$ and upshifting the higher side $(k=K / 2+1, K / 2+2, \ldots, K)$. Figure 7 shows the result of this process. As evident from the figure, the DC null is reduced. Moreover, this frequency shifting enables the fine-adjustment of guard bands.

In the case of OFDM, the number of guard band subcarriers is 157; therefore GFDM system should employ the equivalent bandwidth corresponding to guard bands. The bandwidths corresponding to the guard band and the number of guard band subcarriers for GFDM are computed as follows:

$$
\begin{aligned}
B_{\mathrm{g}} & =157 \times \frac{2640 \mathrm{MHz}}{512} \\
N_{\mathrm{g}, \mathrm{GFDM}} & =\left[\frac{B_{\mathrm{g}}}{2640 \mathrm{MHz} / \mathrm{K}}\right],
\end{aligned}
$$


where $B_{\mathrm{g}}$ and $N_{\mathrm{g}, \mathrm{GFDM}}$ are the bandwidths corresponding to the guard band and the number of guard band subcarriers for GFDM, respectively. The operation [.] represents rounding function. The proposed 1lad GFDM system employs these values.

The mathematical expression of GFDM modulation matrix, $\mathbf{A}$, including the frequency shifting is given below:

$$
\begin{aligned}
\mathbf{A}= & {\left[\begin{array}{llll}
\mathbf{G}_{0} \circ \widetilde{\mathbf{W}}_{K} & \mathbf{G}_{1} \circ \widetilde{\mathbf{W}}_{K} & \cdots & \mathbf{G}_{M-1} \circ \widetilde{\mathbf{W}}_{K}
\end{array}\right] } \\
\in & \mathbb{C}^{N \times K M} \\
\widetilde{\mathbf{W}}_{K}= & {\left[\begin{array}{ccc}
w_{K}^{0 \cdot 0} v_{\Delta f_{0}}^{0} & \cdots & w_{K}^{0 \cdot(K-1)} v_{\Delta f_{K}}^{0} \\
w_{K}^{1 \cdot 0} v_{\Delta f_{0}}^{1} & \cdots & w_{K}^{1 \cdot(K-1)} v_{\Delta f_{K}}^{1} \\
\vdots & \ddots & \vdots \\
w_{K}^{(N-1) \cdot 0} v_{\Delta f_{0}}^{N-1} & \cdots & w_{K}^{(N-1) \cdot(K-1)} v_{\Delta f_{K}}^{N-1}
\end{array}\right] \in \mathbb{C}^{N \times K} } \\
v_{\Delta f_{k}}= & \exp \left(\operatorname{sgn}\left(k-\frac{N_{\mathrm{g}, \mathrm{GFDM}}}{2}\right) j 2 \pi \Delta f\right),
\end{aligned}
$$

where $\operatorname{sgn}(\cdot)$ is sign function which returns the sign of the argument. $\Delta f$ is the frequency shift.

\subsection{GFDM Pilot Symbol Design. In 1lad OFDM PHY, pilot} symbols are mapped onto the specific subcarriers over all time. The pilot symbols are used for the phase derotation at the receiver side. The pilot symbols have to be inserted since received signals cannot be demodulated under existence of the phase rotation. However, in the case of GFDM, if the pilot symbol is transmitted using specific subcarrier over all time, it results in the waste of resources due to the wide subcarrier bandwidth. This paper introduces a scattered pilot structure similar to the one in LTE packet. Figure 8 shows the timefrequency grid of one GFDM symbol. The yellow symbol represents the pilot symbol. The number of pilot symbols is 16 (same as OFDM) and they are mapped onto the lowest subcarrier and the highest subcarrier in a zigzag manner to avoid the ICI effect. Time domain spacing is $\lfloor M / 16\rfloor$ to be widely scattered. The phase tracking performance is shown in Figure 9. The upper plot corresponds to OFDM and the lower plot corresponds to GFDM. As expected, there is no significant difference between these two results.

\section{Interchannel Interference Cancellation}

As described in Section 2.2, the received GFDM symbol can be demodulated by multiplying $\mathbf{A}^{\mathrm{H}}$. However as shown in Figure 4, the ICI effect is significant and it degrades the Bit Error Rate (BER) performance. The ICI reduction schemes for GFDM have been studied and the algorithm named Double Sided Serial Interference Cancellation (DSIC) [34] shows good performance results. However, this ICI reduction scheme is affected by channel equalization errors and noise effects. Moreover, the special blocks for ICI reduction are needed because ICI estimation is done per subcarrier. This complexity comes from the assumption that ICI should be mitigated at the receiver side. The ICI of GFDM is completely

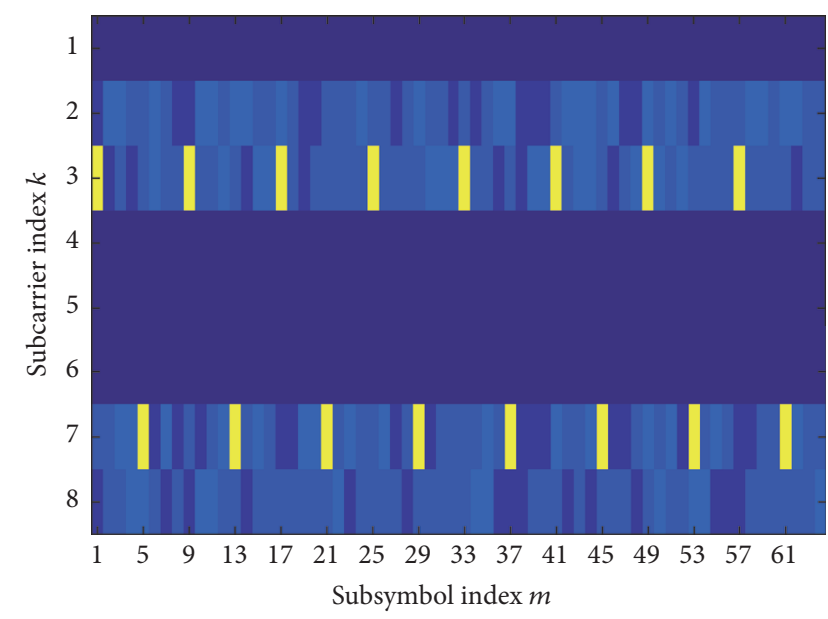

FIGURE 8: Pilot symbol insertion for GFDM symbol $(K=8, M=$ $64)$.
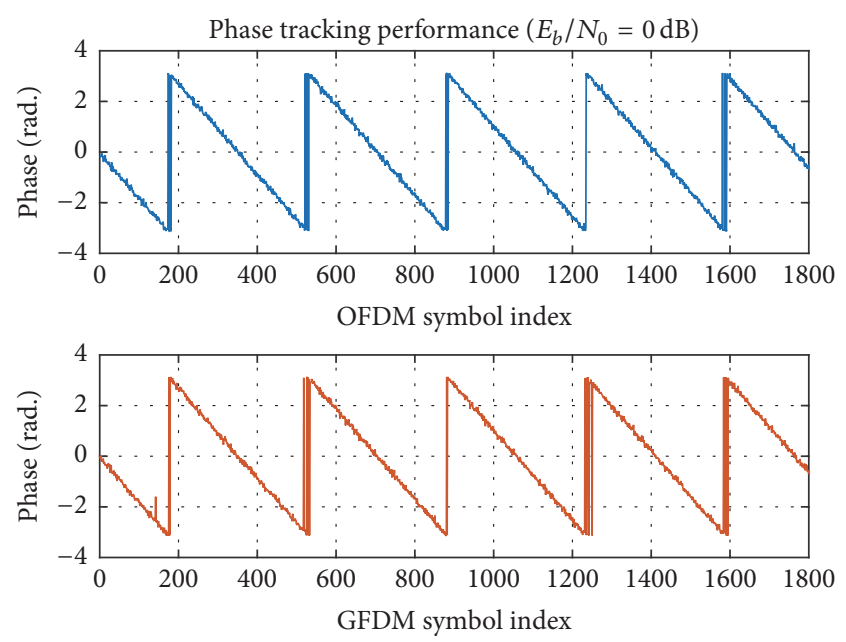

FIGURE 9: Phase tracking performance comparison.

different from the problem in OFDM. This ICI in GFDM is self-interference arose due to subcarrier nonorthogonality. Therefore, the transmitter should be able to estimate the ICI values before the wireless transmission without any noise effects. This paper introduces a transmitter side ICI reduction scheme for GFDM. The proposed ICI reduction block is illustrated as shown in Figure 10.

$\mathbf{d}$ and $\overline{\mathbf{d}}$ are original QAM modulated symbol and ICI subtracted symbol, respectively. In addition, iterative calculation is desired in order to reduce ICI effects effectively because ICI subtraction leads to another ICI. This ICI reduction block is constructed with GFDM modulator, GFDM demodulator, and adder. According to the fact that the device which has GFDM should have both modulator and demodulator, this ICI reduction block does not require special additional block.

\section{Simulation Study}

5.1. Deriving $M$ and $K$ for Improved Performance. As presented in previous sections, 1lad GFDM system performance 


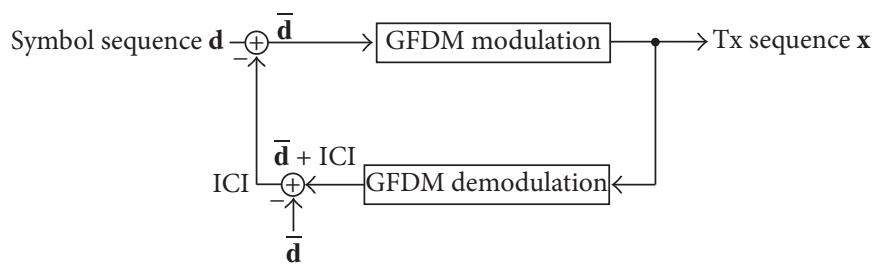

FIgURE 10: Transmitter side ICI reduction brock diagram.

TABLE 2: IEEE 802.11ad GFDM parameters.

\begin{tabular}{lc}
\hline Parameter & Value \\
\hline $\begin{array}{l}\text { Number of subcarriers } K \\
\text { Number of subsymbols } \\
M\end{array}$ & $4,6,8,16,32,128$ \\
Number of pilot symbols & $\lfloor 512 / K\rfloor$ \\
Number of DC & 16 \\
subcarriers & 1 \\
Number of guard band \\
subcarriers \\
$\begin{array}{lc}\text { GFDM sample rate } & N_{\mathrm{g}, \mathrm{GFDM}} \\
\text { GFDM symbol period } & 2640 \mathrm{MHz} \\
\text { Cyclic prefix duration } & 48.4 \mathrm{~ns}+(512-K \times M) / 2640 \mathrm{MHz} \\
\text { Channel model } & 11 \text { ad cubicle LOS/NLOS (far laptop) [35] } \\
\text { Carrier frequency offset } & 0-10 \mathrm{MHz}(\text { random generated) } \\
\text { Pulse shaping filter } & \text { Root Raised Cosine } \\
\text { Roll-off factor } \alpha & 0.1,0.2,0.3,0.4 \\
\text { Modulation and coding } \\
\text { scheme } \\
\text { Number of ICI canceler } \\
\text { iterations }\end{array}$ \\
\hline
\end{tabular}

is evaluated in terms of PAPR and BER. The simulation parameters are provided in Table 2 . The second term of the cyclic prefix is for the symbol length adjustment when $K \times M \neq 512$. For the simple derivation, GFDM with $K$ subcarriers and $M$ subsymbols is derived as $(K, M)$-GFDM hereafter.

5.1.1. PAPR Comparison. Figure 11 shows the complementary cumulative distribution function (CCDF) of PAPR. This simulation assumes QPSK transmission with the roll-off factor $\alpha=0.1$. Although the PAPR value changes if other modulation schemes are employed, relativity is not changed. In the view point of PAPR, $(4,128)$-GFDM achieves the best performance due to the small number of subcarriers. However, in this case, the number of guard band subcarriers is 1 ; that is, $N_{\mathrm{g}, \mathrm{GFDM}}=1$. Therefore, the effective number of subcarriers is only two. It means that one $(4,128)$-GFDM symbol can only transmit $2 \times 128=256$ symbols. To make matters worth, it contains pilot symbols. Consequently, $(4,128)$ GFDM can only transmit 240 data symbols with one GFDM symbol. Therefore $(4,128)-G F D M$ degrades throughput performance. On the other hand, in $(6,85)$-GFDM and $(8,64)$ GFDM case, they still show good PAPR performance. From

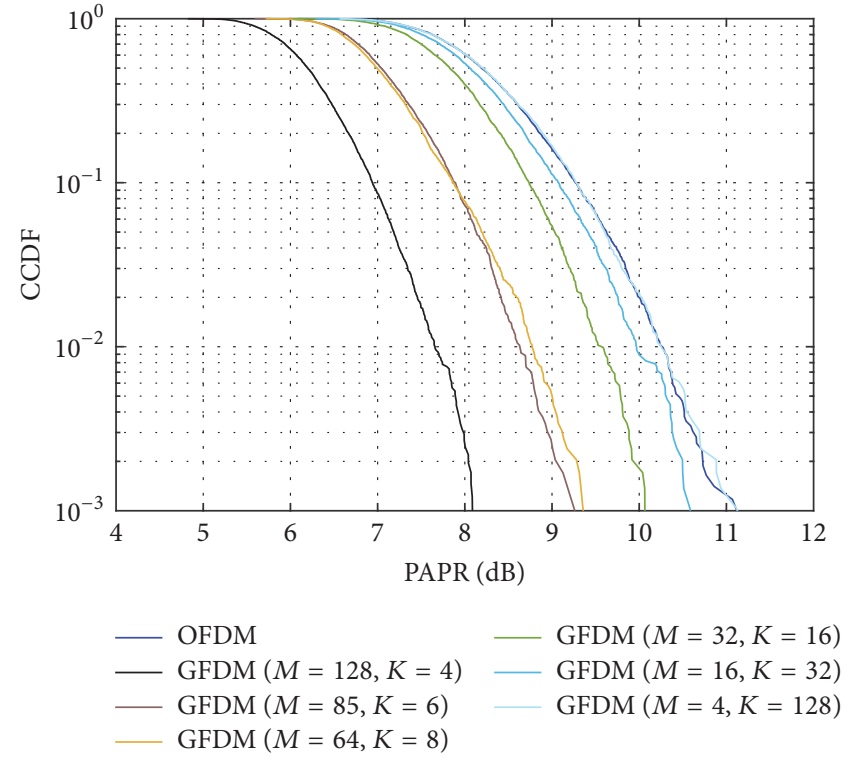

FIGURE 11: PAPR comparison between OFDM and GFDM (QPSK, $\alpha=0.1$ ).

the throughput point of view, 324 and 240 data symbols can be transmitted using $(6,85)$-GFDM and $(8,64)$-GFDM, respectively. Therefore $(6,85)$-GFDM can achieve the best PAPR performance while it maintains throughput performance. If there is no guard band insertion, it is obvious that the throughput performances are same between all configurations and PAPR performance can be improved by decreasing the number of subcarriers. Therefore only from the view point of PAPR, SC is the best solution. However, SC is strongly affected by the multipath effect. Based on these issues, this paper concludes that $(6,85)$-GFDM is the best in order to maintain high throughput as well as good PAPR performance. Hereafter, performance evaluation focuses the $(6,85)$-GFDM.

Figure 12 shows PAPR dependency on roll-off factor $\alpha$. As shown in Figure 12, 16QAM and 64QAM show no significant difference, while QPSK shows remarkable performance improvement. If $\alpha=0.4$ is considered, PAPR performance reaches the performance of $(4,128)$-GFDM.

5.1.2. BER Performance Comparison. Figure 13 compares the BER performance of OFDM and several GFDM configuration systems in LOS environment. As shown in Figure 13, 


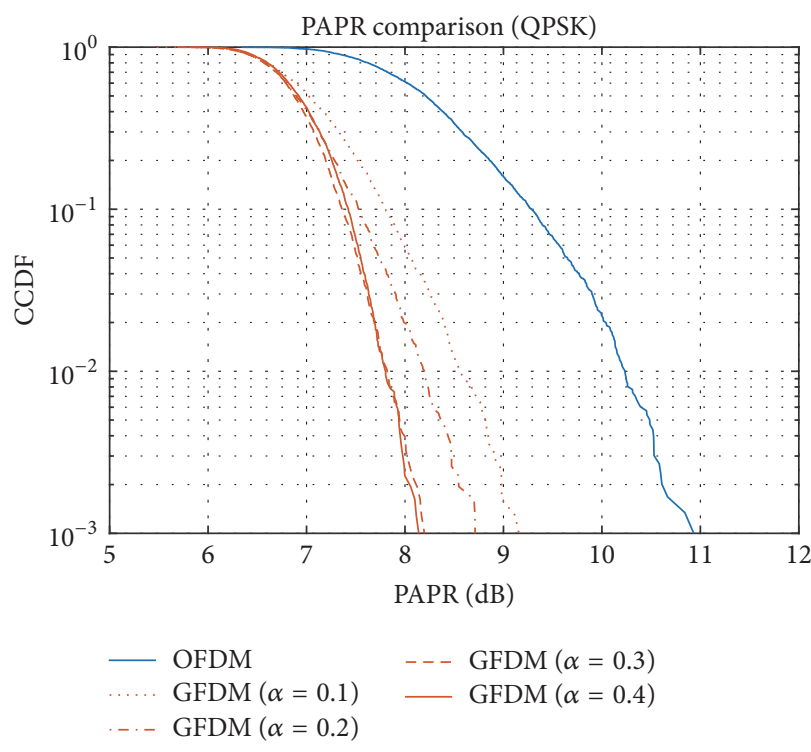

(a) QPSK

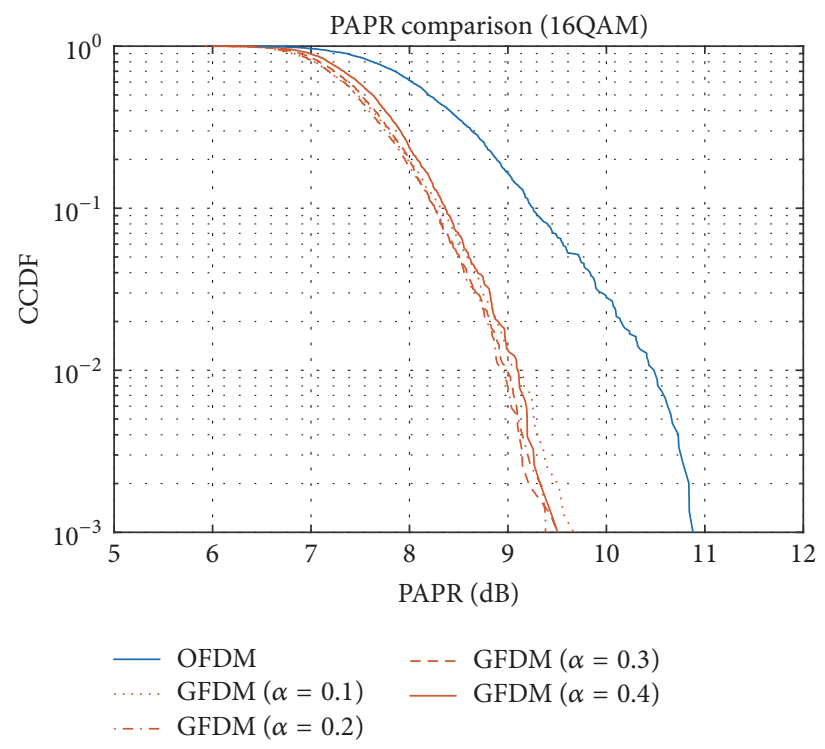

(b) 16QAM

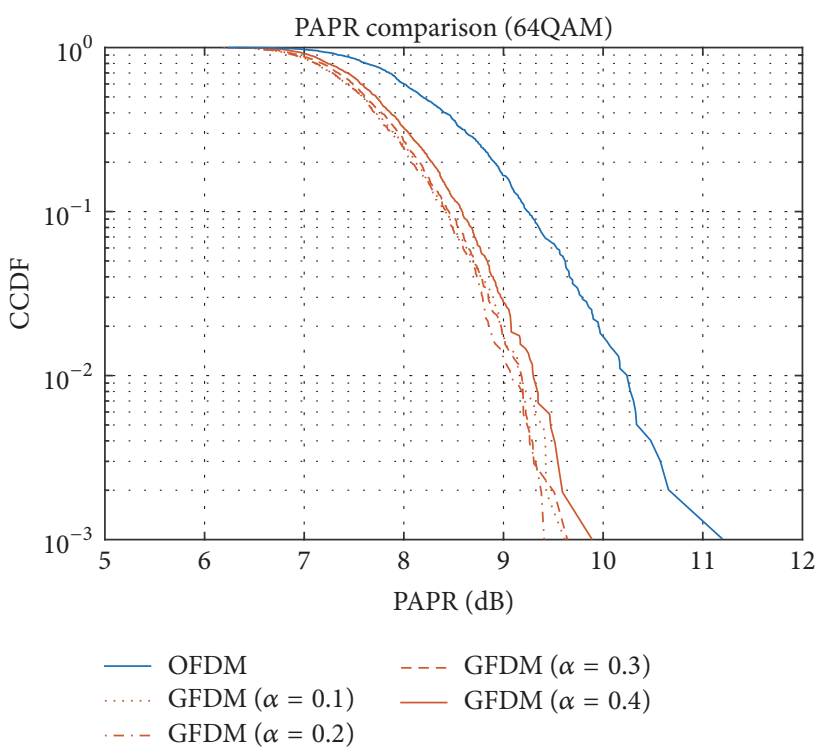

(c) $64 \mathrm{QAM}$

FIGURE 12: PAPR dependency on roll-off factor.

there is no significant difference in the BER values for different iterations. Two iterations are sufficient to achieve good BER performance. In addition, the OFDM and GFDM show similar BER performances in the best case. These performance results indicate that $(6,85)-G F D M$ can reduce PAPR up to $3 \mathrm{~dB}$ without compromising the system throughput.

Figure 14 shows the BER performance in NLOS environment. It can be seen that the GFDM is significantly better than the OFDM system. This is because of the channel equalization error effect becoming more dominant in NLOS conditions. This error causes frequency selectivity. Since OFDM uses narrow band subcarriers, this effect degrades BER performance. On the other hand, GFDM is relatively more robust to this effect because of the wide bandwidths of the subcarriers.

\section{Computational Complexity}

Theoretically, GFDM modulation and demodulation can be realized by simply multiplying the modulation matrix $\mathbf{A}$ as described in Section 2.2. However this simple approach requires high computational costs, that is, the large number of complex multiplications. In addition, constructing modulation matrix $\mathbf{A}$ also requires high computational costs because it is constructed with the Hadamard product of complex 


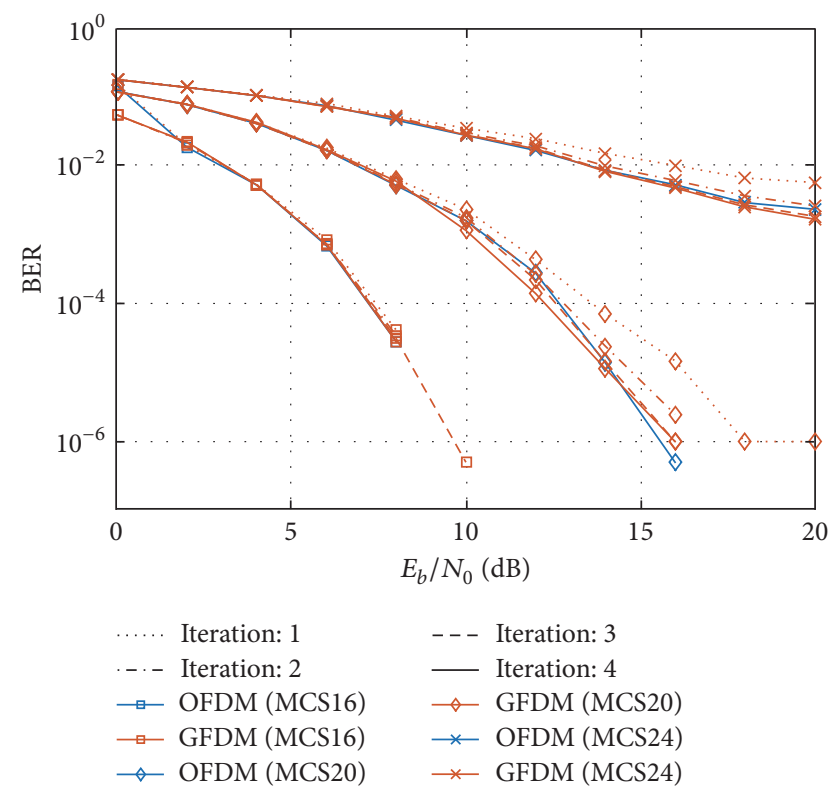

FIGURE 13: BER comparison between OFDM and GFDM (MCS 16, 20 , and 24, $\alpha=0.1$, LOS).

matrix. In order to reduce the number of complex multiplications, modulation and demodulation blocks should be reconfigured so that FFT can be used. Equation (3) can be rewritten as follows:

$$
\begin{aligned}
x[n] & =\sum_{k=0}^{K-1} \sum_{m=0}^{M-1} d_{k, m} \widetilde{g}[n-m K] \exp \left(j 2 \pi \frac{n k}{K}\right) \\
& =\sum_{m=0}^{M-1} \tilde{g}[n-m K] \underbrace{\sum_{k=0}^{K-1} d_{k, m} \exp \left(j 2 \pi \frac{n k}{K}\right)}_{\text {IDFT }} \\
& =\sum_{m=0}^{M-1} \tilde{g}[n-m K] D_{m}[n \bmod K],
\end{aligned}
$$

where $D_{m}[n \bmod K]$ is IDFT of $d_{k, m}$. Since $n=0,1, \ldots$, $K M-1, D_{m}[n]$ becomes circular repetition sequence. Equation (7) shows that $x[n]$ can be computed using IDFT and summing up the filtered $M$ sequences. The total number of the complex multiplications involved in GFDM modulator is $(K \log K($ IFFT $)+M K($ Filtering $)) \times M=(\log K+M) M K$. follows:

Similarly, GFDM demodulation can be described as

$$
\begin{aligned}
\widehat{d}_{k^{\prime}, m^{\prime}} & =\sum_{n=0}^{K M-1} \tilde{g}\left[n-m^{\prime} K\right] x[n] \exp \left(-j 2 \pi \frac{n k^{\prime}}{K}\right) \\
= & \sum_{l=0}^{M-1} \sum_{n=l K}^{(l+1) K-1} \tilde{g}\left[n-m^{\prime} K\right] x[n] \exp \left(-j 2 \pi \frac{n k^{\prime}}{K}\right) \\
= & \sum_{l=0}^{M-1} \sum_{n^{\prime}=0}^{K-1} \tilde{g}\left[n^{\prime}+l K-m^{\prime} K\right] x\left[n^{\prime}+l K\right] \exp (-j 2 \pi
\end{aligned}
$$

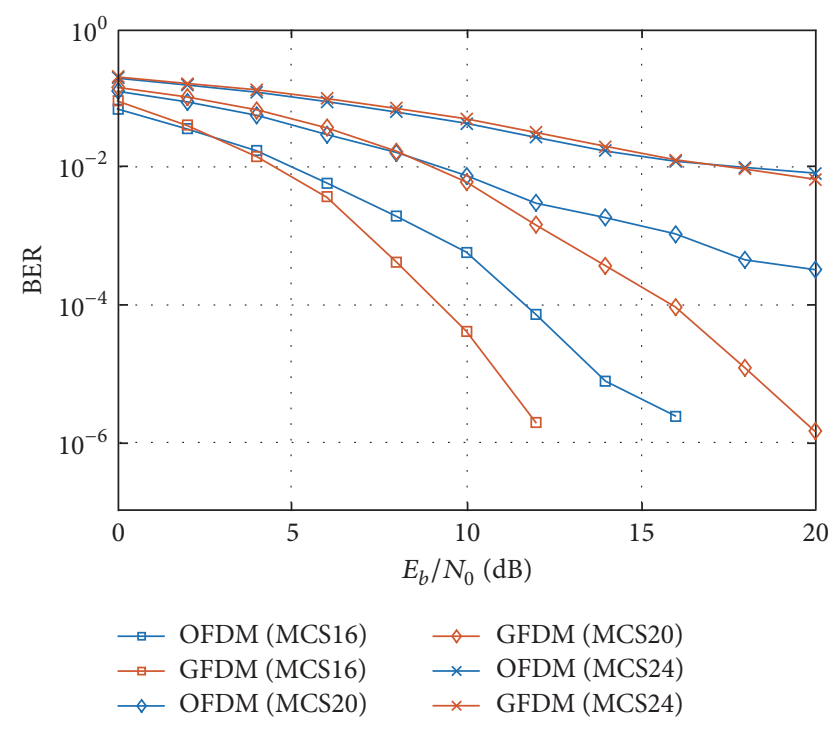

FIGURE 14: BER comparison between OFDM and GFDM (MCS 16, 20 , and $24, \alpha=0.1$, NLOS).

$$
\begin{aligned}
& \left.\cdot \frac{k^{\prime}\left(n^{\prime}+l K\right)}{K}\right) \\
& =\underbrace{\sum_{l=0}^{M-1} \tilde{g}\left[n^{\prime}+l K-m^{\prime} K\right] x\left[n^{\prime}+l K\right] \sum_{n^{\prime}=0}^{K-1} \exp (-j 2 \pi}_{\text {Correlation receiver }} \\
& \left.\cdot \frac{k^{\prime} n^{\prime}}{K}\right)=\underbrace{\sum_{n^{\prime}=0}^{K-1} D_{m^{\prime}}\left[n^{\prime}\right] \exp \left(-j 2 \pi \frac{k^{\prime} n^{\prime}}{K}\right)}_{\text {DFT }} .
\end{aligned}
$$

Thus, the total number of the complex multiplications involved in GFDM demodulator is $(K \log K(\mathrm{FFT})+$ $M K($ Filtering $)) \times M=(\log K+M) M K$.

The modified modulation and demodulation blocks according to these equations are as illustrated in Figure 15.

Originally, the computational costs of matrix multiplication are $M^{2} K^{2}$. Therefore, the computational cost of this modulation/demodulation blocks becomes $(\log K+M) M K /$ $M^{2} K^{2}=(\log K+M) / M K=0.17$ for $(K, M)=(6,85)$. Thus, the proposed structure can reduce about $83 \%$ of the computational cost. In [36], the proposed GFDM transceiver architecture cannot apply the FFT/IFFT blocks. In contrast, our proposed architecture can utilize the FFT/IFFT blocks and it can drastically reduce the computational cost.

\section{Concluding Remarks and Future Work}

This study has investigated the methodology for adapting GFDM to one of the most successful standards, that is, IEEE 802.11ad. It shows that the proposed GFDM frame structure is fully in compliance with the existing IEEE 802.11ad. Moreover, this study extends the ICI reduction technique to be suitable for IEEE 802.11ad wireless systems. The intensive 


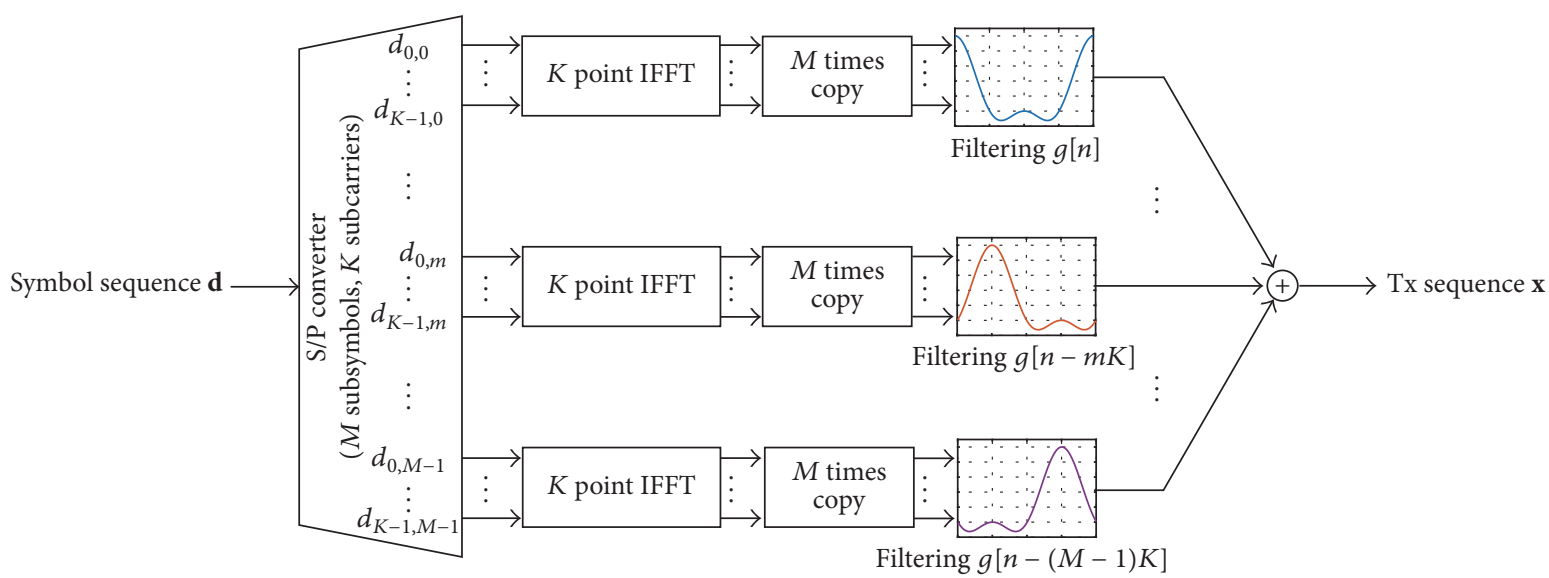

(a) GFDM modulator

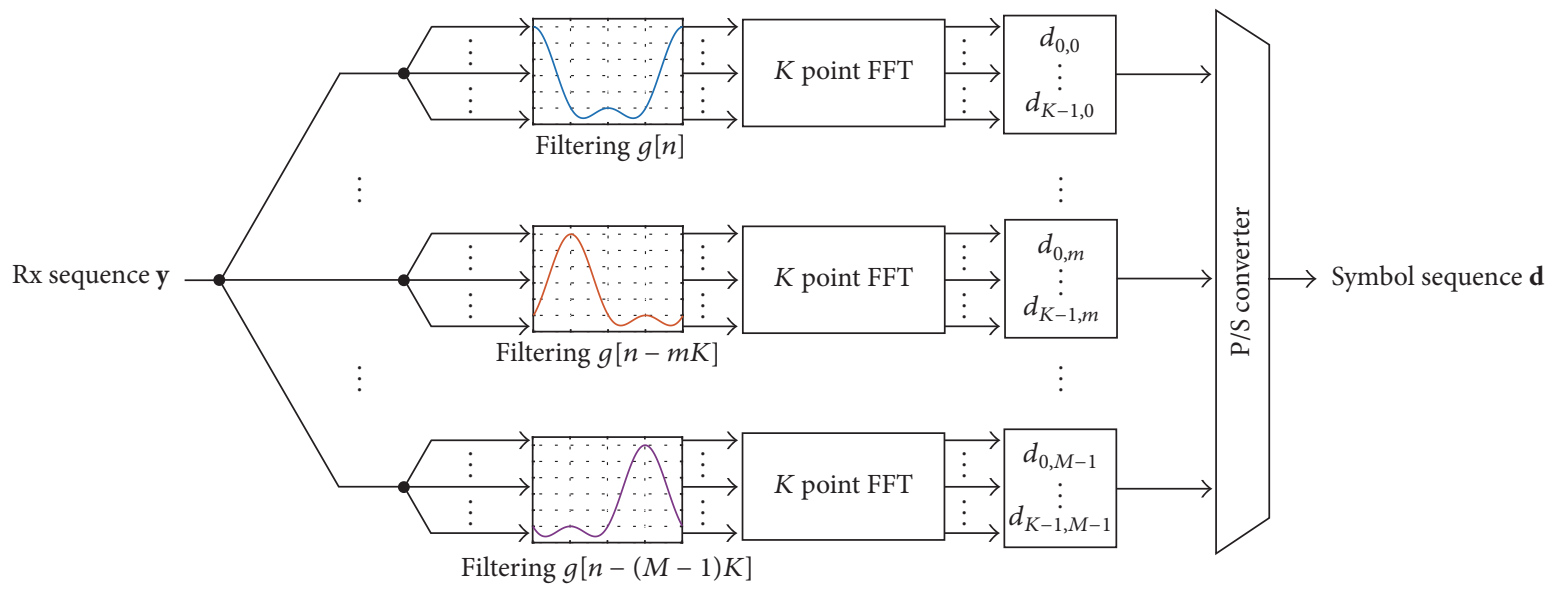

(b) GFDM demodulator

FIGURE 15: GFDM modulator and demodulator block.

performance evaluation results in this paper reveal that $(6,85)$-GFDM could achieve good PAPR and throughput performance simultaneously. The proposed efficient GFDM modulation/demodulation block can reduce computational costs by about $83 \%$. The proposed method is applicable for other mmWave standards in IEEE 802.11ay and 5G mmWave cellular networks.

As a future research direction, it is possible to conduct more intensive performance evaluation considering the radio frequency (RF) impairments including power amplifier nonlinearity, IQ imbalance, phase noises, and so forth.

\section{Competing Interests}

The authors declare that they have no competing interests.

\section{Acknowledgments}

This work was supported by Intel Next Generation and Standards (NGS) funds and also by National Research Foundation of Korea (NRF Korea) under Grant 2016R1C1B1015406.

\section{References}

[1] J. Kim, Y. Tian, S. Mangold, and A. F. Molisch, "Joint scalable coding and routing for $60 \mathrm{GHz}$ real-time live HD video streaming applications," IEEE Transactions on Broadcasting, vol. 59, no. 3, pp. 500-512, 2013.

[2] J. Kim, Y. Tian, S. Mangold, and A. F. Molisch, "Quality-aware coding and relaying for $60 \mathrm{GHz}$ real-time wireless video broadcasting," in Proceedings of the IEEE International Conference on Communications (ICC '13), pp. 5148-5152, Budapest, Hungary, June 2013.

[3] Y. Azar, G. N. Wong, K. Wang et al., "28 GHz propagation measurements for outdoor cellular communications using steerable beam antennas in New York city," in Proceedings of the IEEE International Conference on Communications (ICC '13), pp. 5143-5147, Budapest, Hungary, June 2013.

[4] H. Zhao, R. Mayzus, S. Sun et al., " 28 GHz millimeter wave cellular communication measurements for reflection and penetration loss in and around buildings in New York city," in Proceedings of the IEEE International Conference on Communications (ICC '13), pp. 5163-5167, Budapest, Hungary, June 2013.

[5] T. S. Rappaport, F. Gutierrez, E. Ben-Dor, J. N. Murdock, Y. Qiao, and J. I. Tamir, "Broadband millimeter-wave propagation 
measurements and models using adaptive-beam antennas for outdoor urban cellular communications," IEEE Transactions on Antennas and Propagation, vol. 61, no. 4, pp. 1850-1859, 2013.

[6] W. Roh, J.-Y. Seol, J. Park et al., "Millimeter-wave beamforming as an enabling technology for $5 \mathrm{G}$ cellular communications: theoretical feasibility and prototype results," IEEE Communications Magazine, vol. 52, no. 2, pp. 106-113, 2014.

[7] S. Shepherd, J. Orriss, and S. Barton, "Asymptotic limits in peak envelope power reduction by redundant coding in orthogonal frequency-division multiplex modulation," IEEE Transactions on Communications, vol. 46, no. 1, pp. 5-10, 1998.

[8] T. Jiang, M. Guizani, H.-H. Chen, W. Xiang, and Y. Wu, "Derivation of PAPR distribution for OFDM wireless systems based on extreme value theory," IEEE Transactions on Wireless Communications, vol. 7, no. 4, pp. 1298-1305, 2008.

[9] T. Jiang and Y. Wu, "An overview: peak-to-average power ratio reduction techniques for OFDM signals," IEEE Transactions on Broadcasting, vol. 54, no. 2, pp. 257-268, 2008.

[10] J. Armstrong, "Peak-to-average power reduction for OFDM by repeated clipping and frequency domain filtering," Electronics Letters, vol. 38, no. 5, pp. 246-247, 2002.

[11] H. Ochiai and H. Imai, "On clipping for peak power reduction of OFDM signals," in Proceedings of the IEEE Global Telecommunications Conference (GLOBECOM '00), San Francisco, Calif, USA, December 2000.

[12] Y.-C. Wang and Z.-Q. Luo, "Optimized iterative clipping and filtering for PAPR reduction of OFDM signals," IEEE Transactions on Communications, vol. 59, no. 1, pp. 33-37, 2011.

[13] X. Wang, T. T. Tjhung, and C. S. Ng, "Reduction of peak-toaverage power ratio of OFDM system using a companding technique," IEEE Transactions on Broadcasting, vol. 45, no. 3, pp. 303-307, 1999.

[14] X. Huang, J. Lu, J. Zheng, K. B. Letaief, and J. Gu, "Companding transform for reduction in peak-to-average power ratio of OFDM signals," IEEE Transactions on Wireless Communications, vol. 3, no. 6, pp. 2030-2039, 2004.

[15] T. Jiang, Y. Yang, and Y.-H. Song, "Exponential companding technique for PAPR reduction in OFDM systems," IEEE Transactions on Broadcasting, vol. 51, no. 2, pp. 244-248, 2005.

[16] S. H. Müller and J. B. Huber, "OFDM with reduced peak-toaverage power ratio by optimum combination of partial transmit sequences," Electronics Letters, vol. 33, no. 5, pp. 368-369, 1997.

[17] S.-J. Ku, C.-L. Wang, and C.-H. Chen, "A reduced-complexity PTS-based PAPR reduction scheme for OFDM systems," IEEE Transactions on Wireless Communications, vol. 9, no. 8, pp. 2455-2460, 2010.

[18] L. Yang, R. S. Chen, Y. M. Siu, and K. K. Soo, "PAPR reduction of an OFDM signal by use of PTS with low computational complexity," IEEE Transactions on Broadcasting, vol. 52, no. 1, pp. 83-86, 2006.

[19] R. W. Bäuml, R. F. H. Fischer, and J. B. Huber, "Reducing the peak-to-average power ratio of multicarrier modulation by selected mapping," Electronics Letters, vol. 32, no. 22, pp. 20562057, 1996.

[20] S. H. Han and J. H. Lee, "Modified selected mapping technique for PAPR reduction of coded OFDM signal," IEEE Transactions on Broadcasting, vol. 50, no. 3, pp. 335-341, 2004.

[21] C.-P. Li, S.-H. Wang, and C.-L. Wang, "Novel low-complexity SLM schemes for PAPR reduction in OFDM systems," IEEE Transactions on Signal Processing, vol. 58, no. 5, pp. 2916-2921, 2010.
[22] B. Farhang-Boroujeny, "OFDM versus filter bank multicarrier," IEEE Signal Processing Magazine, vol. 28, no. 3, pp. 92-112, 2011.

[23] Z. Kollár and P. Horváth, "PAPR reduction of FBMC by clipping and its iterative compensation," Journal of Computer Networks and Communications, vol. 2012, Article ID 382736, 11 pages, 2012.

[24] N. Van Der Neut, B. T. Maharaj, F. H. De Lange, G. Gonzalez, F. Gregorio, and J. Cousseau, "PAPR reduction in FBMC systems using a smart gradient-project active constellation extension method," in Proceedings of the 21st International Conference on Telecommunications (ICT '14), pp. 134-139, Lisbon, Portugal, May 2014.

[25] G. Fettweis, M. Krondorf, and S. Bittner, "GFDM-generalized frequency division multiplexing," in Proceedings of the IEEE Vehicular Technology Conference (VTC '09), Barcelona, Spain, April 2009.

[26] N. Michailow, M. Matthe, I. S. Gaspar et al., "Generalized frequency division multiplexing for 5th generation cellular networks," IEEE Transactions on Communications, vol. 62, no. 9, pp. 3045-3061, 2014.

[27] A. Y. Panah, R. G. Vaughan, and R. W. Heath Jr., "Optimizing pilot locations using feedback in OFDM systems," IEEE Transactions on Vehicular Technology, vol. 58, no. 6, pp. 2803-2814, 2009.

[28] E. Bedeer, O. A. Dobre, M. H. Ahmed, and K. E. Baddour, "Rateinterference Tradeoff in OFDM-based cognitive radio systems," IEEE Transactions on Vehicular Technology, vol. 64, no. 9, pp. 4292-4298, 2015.

[29] S. Bokharaiee, H. H. Nguyen, and E. Shwedyk, "Blind spectrum sensing for OFDM-based cognitive radio systems," IEEE Transactions on Vehicular Technology, vol. 60, no. 3, pp. 858-871, 2011.

[30] J. Ylioinas and M. Juntti, "Iterative joint detection, decoding, and channel estimation in turbo-coded MIMO-OFDM," IEEE Transactions on Vehicular Technology, vol. 58, no. 4, pp. 17841796, 2009.

[31] C. Shin, R. W. Heath Jr., and E. J. Powers, "Blind channel estimation for MIMO-OFDM systems," IEEE Transactions on Vehicular Technology, vol. 56, no. 2, pp. 670-685, 2007.

[32] H. H. Ma and J. K. Wolf, "On tail biting convolutional codes," IEEE Transactions on Communications, vol. 34, no. 2, pp. 104111, 1986.

[33] IEEE P802.11 TGad, "PHY/MAC Complete Proposal Specification," May 2010.

[34] R. Datta, N. Michailow, M. Lentmaier, and G. Fettweis, "GFDM interference cancellation for flexible cognitive radio phy design," in Proceedings of the 76th IEEE Vehicular Technology Conference (VTC '12), Quebec, Canada, September 2012.

[35] IEEE P802.11 TGad, "Channel Models for $60 \mathrm{GHz}$ WLAN Systems," May 2010.

[36] N. Michailow, I. Gaspar, S. Krone, M. Lentmaier, and G. Fettweis, "Generalized frequency division multiplexing: analysis of an alternative multi-carrier technique for next generation cellular systems," in Proceedings of the 9th International Symposium on Wireless Communication Systems (ISWCS '12), pp. 171-175, August 2012. 


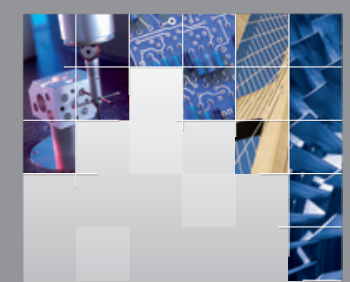

\section{Enfincering}
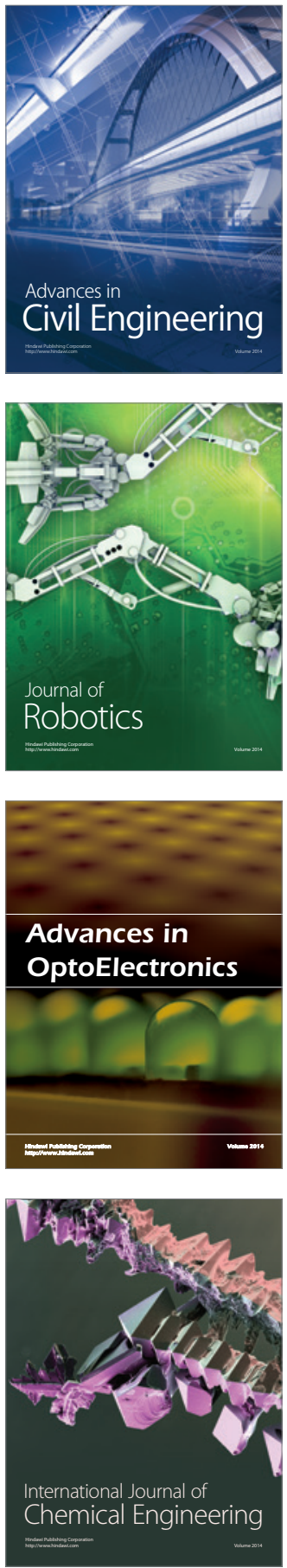

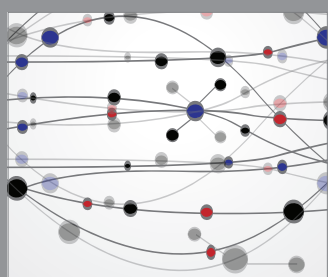

The Scientific World Journal

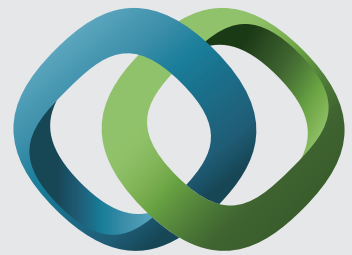

\section{Hindawi}

Submit your manuscripts at

http://www.hindawi.com
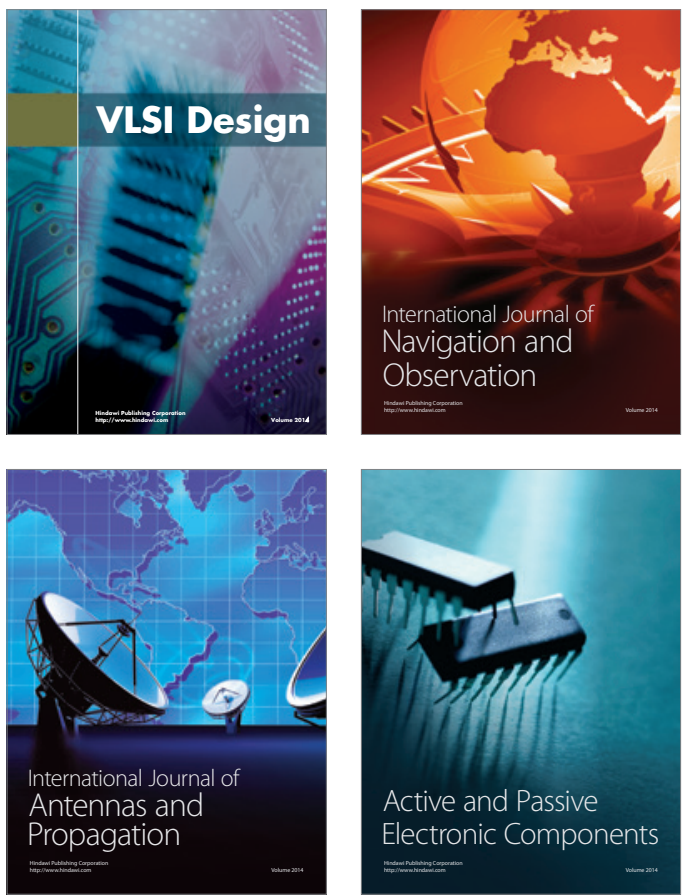
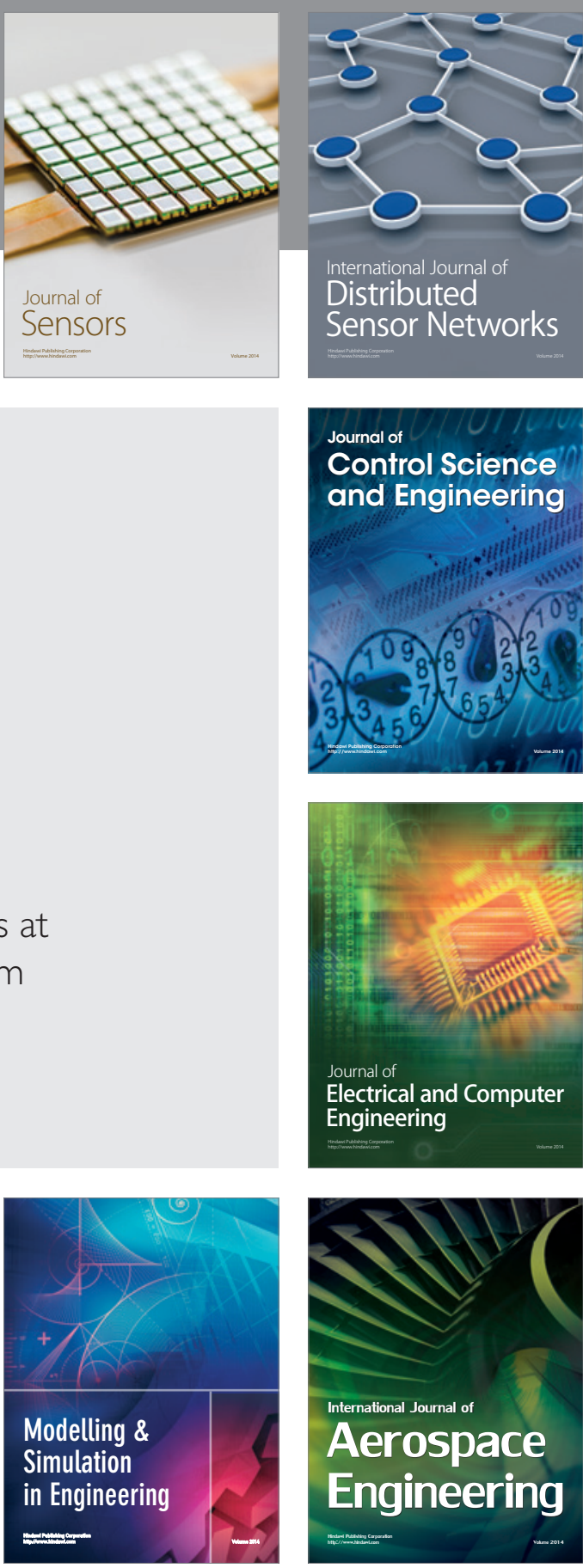

International Journal of

Distributed

Sensor Networks

Journal of

Control Science

and Engineering
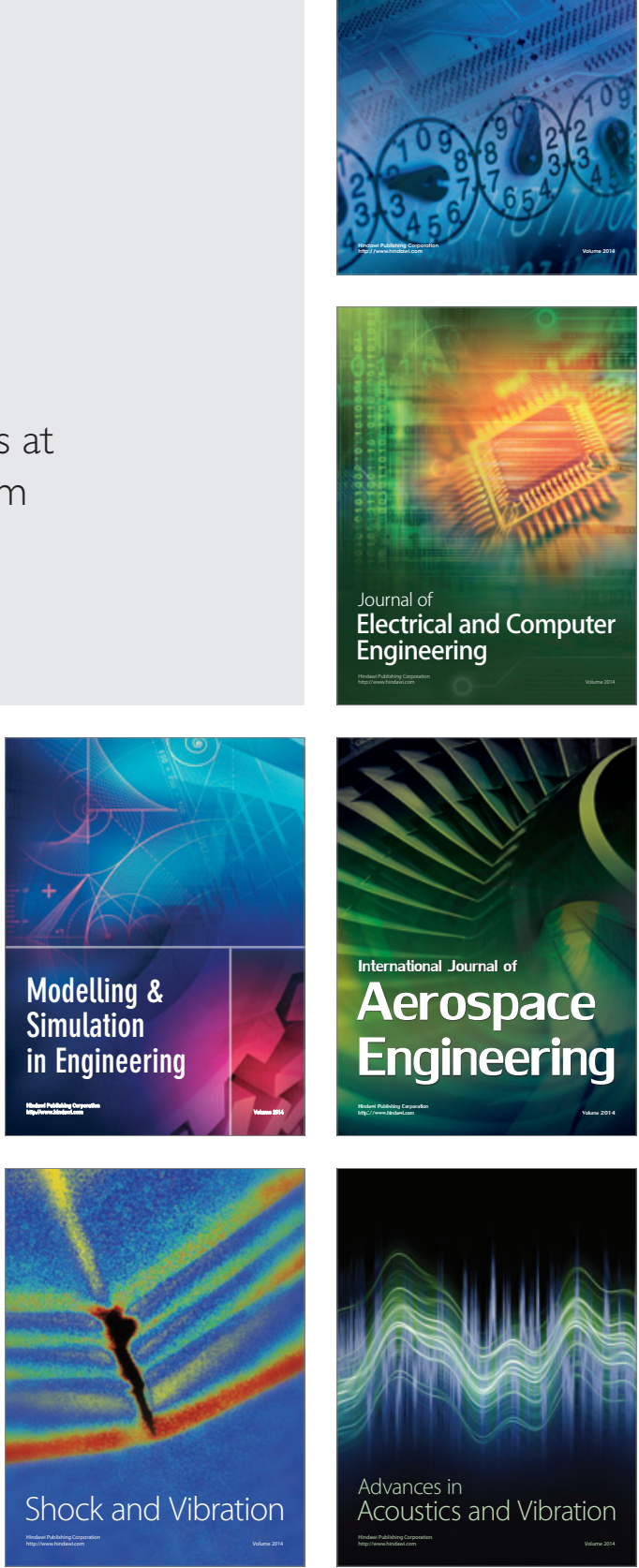